\title{
An approach to fast fits of the unintegrated gluon density
}

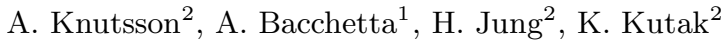 \\ 1- Jefferson Laboratory, Theory Center, 12000 Jefferson Av. Newport News, VA, 23606, USA \\ 2- DESY, Notkestrasse 85, 22603 Hamburg, Germany
}

\begin{abstract}
An approach to fast fits of the unintegrated gluon density has been developed and used to determine the unintegrated gluon density by fits to deep inelastic scatting di-jet data from HERA. The fitting method is based on the determination of the parameter dependence by help of interpolating between grid points in the parameter-observable space before the actual fit is performed.
\end{abstract}

\section{Introduction}

The substructure of the proton is parameterized by parton density functions (PDFs). In perturbative QCD the PDFs are given by solutions of integral equations, for which the initial input distributions have to be determined by fits. It turns out that, for exclusive final states, it is not statistically efficient to tune Monte Carlo event generators (MC) by sequential calls of the generator together with a minimisation program. Motivated by [1], we use an alternative fitting method, which is based on producing a grid in parameterobservable space. This allows the parameter dependence to be determined by polynomial interpolation before the fit is performed, which significantly reduce the time for performing the fit.

Here we determine the parameters in the starting distribution of the unintegrated gluon density function (uGDF) by using the CASCADE Monte Carlo event generator [2]. The fit is performed to low $Q^{2}$ di-jet data from the H1 experiment [3].

\section{The Unintegrated Gluon Density}

The starting distribution of the unintegrated gluon density is parameterized as

$$
A_{0}\left(x, k_{t}\right)=N x^{-B}(1-x)^{C} \exp \left(k_{t}-\mu\right)^{2} / \sigma^{2}
$$

where $x$ is the longitudinal momentum fraction of the proton carried by the gluon and $k_{t}$ its transverse momentum. Here we use the fitting method to determine the normalisation, $N$, the small $x$ behaviour, $B$, and the shift, $\mu$, of the Gaussian for the transverse momentum of the non-perturbative gluon. The parameters $C$ and $\sigma$, is kept fixed at $C=4$ and $\sigma=2$.

The starting distribution is evolved to higher scales by gluon emissions according to the CCFM evolution equation which impose angular ordering of the emitted gluons.

\section{The Fitting Method}

In the first step of the fitting procedure we build up a grid of $\mathrm{MC}$ predictions in the parameter space $\left(p_{1}, p_{2}, \ldots, p_{n}\right)$ for each of the observables $X$. 
Secondly, we use the grid to describe the parameter-observable space analytically, by using a polynomial of the form

$$
X\left(p_{1}, p_{2}, . ., p_{n}\right)=A_{0}+\sum_{i=1}^{n} B_{i} p_{i}+\sum_{i=1}^{n} C_{i} p_{i}^{2}+\sum_{i=1}^{n-1} \sum_{j=i+i}^{n} D_{i j} p_{i} p_{j}+\text { H.O. }
$$

It is here possible to minimise $\chi^{2}$ with singular value decomposition (SVD)[4], since the coefficients which are to be determined $\left(A_{0}, B_{1}, \ldots\right)$ are mapping the MC grid on the observables in an over determined system of linear equations. This is done separately for each of the MC predicted data points.

In order to account for correlations between parameters the form of the polynomial has to be of at least second order. In the presented fit we use a third order polynomial, which gives a significantly better description of the parameter space.

Having determined the polynomial describing the parameter space, we can fit the parameters $p_{1}, p_{2}, \ldots$ This is done by applying a $\chi^{2}$ minimisation to

$$
\chi^{2}=\sum \frac{\left(X_{k, \text { poly }}-X_{k, \text { data }}\right)^{2}}{\left(\delta X_{k, \text { poly }}-\delta X_{k, \text { data }}\right)^{2}}
$$

where the sum runs over all data points, $k . X_{k, \text { data }}$ is the measured data point, with the corresponding experimental error $\delta X_{k, \text { data }}$, and $X_{k, \text { poly }}$ the polynomial prediction, with the error $\delta X_{k, p o l y}$ calculated from the individual errors of the fitted coefficients by using the covariance matrix. To perform this last step we use MINUIT since the dependence on parameters $p_{1}, p_{2}, \ldots$ is non-linear.

The method turns out to be very time efficient in particular since the MC grid points are generated simultaneously and instead of fitting MC to data we perform a fit of the polynomials, which is much faster due to the fact that the event generation already is performed. The method also allows for very fast refitting if one, for example, wants to study the exclusion of some experimental data points.

\section{The Experimental Data}

The measurement used for the fit was made by $\mathrm{H} 1[3]$ at $\sqrt{s}=318 \mathrm{GeV}$ within the kinematic range $5<Q^{2}<100 \mathrm{GeV}^{2}, 10^{-4}<x_{B j}<10^{-2}$ and $0.1<y<0.7$. Jets are defined in the $\gamma \mathrm{p}$ rest frame with the inclusive $k_{t}$-algorithm, and required to fulfill $E_{T}^{H C M}>5 \mathrm{GeV}$ and $-1<\eta^{L A B}<2.5$, where $E_{T}^{H C M}$ is the transverse momentum in the hadronic center of mass frame and $\eta^{L A B}$ is the pseudorapidity in the laboratory frame. Events with at least two jets fulfilling these requirements were analysed. The total di-jet cross-section was measured as a function of $\Delta$ which gives an additional restriction on the hardest di-jet according to $E_{T}^{H C M}>5+\Delta \mathrm{GeV}$. The cross-section were made double differential by binning in $x_{B j}$ and $Q^{2}$.

\section{Results}

The result of the fit to the $\mathrm{H} 1$ di-jet data is shown in Fig. 1 together with the data as well as three already existing uGDFs. The parameter values determined from the fit are $N=0.28$, $B=0.25$ and $\mu=3.0$. Scanning $\chi^{2}$ as a function of these parameters, as shown in Fig. 2 , 


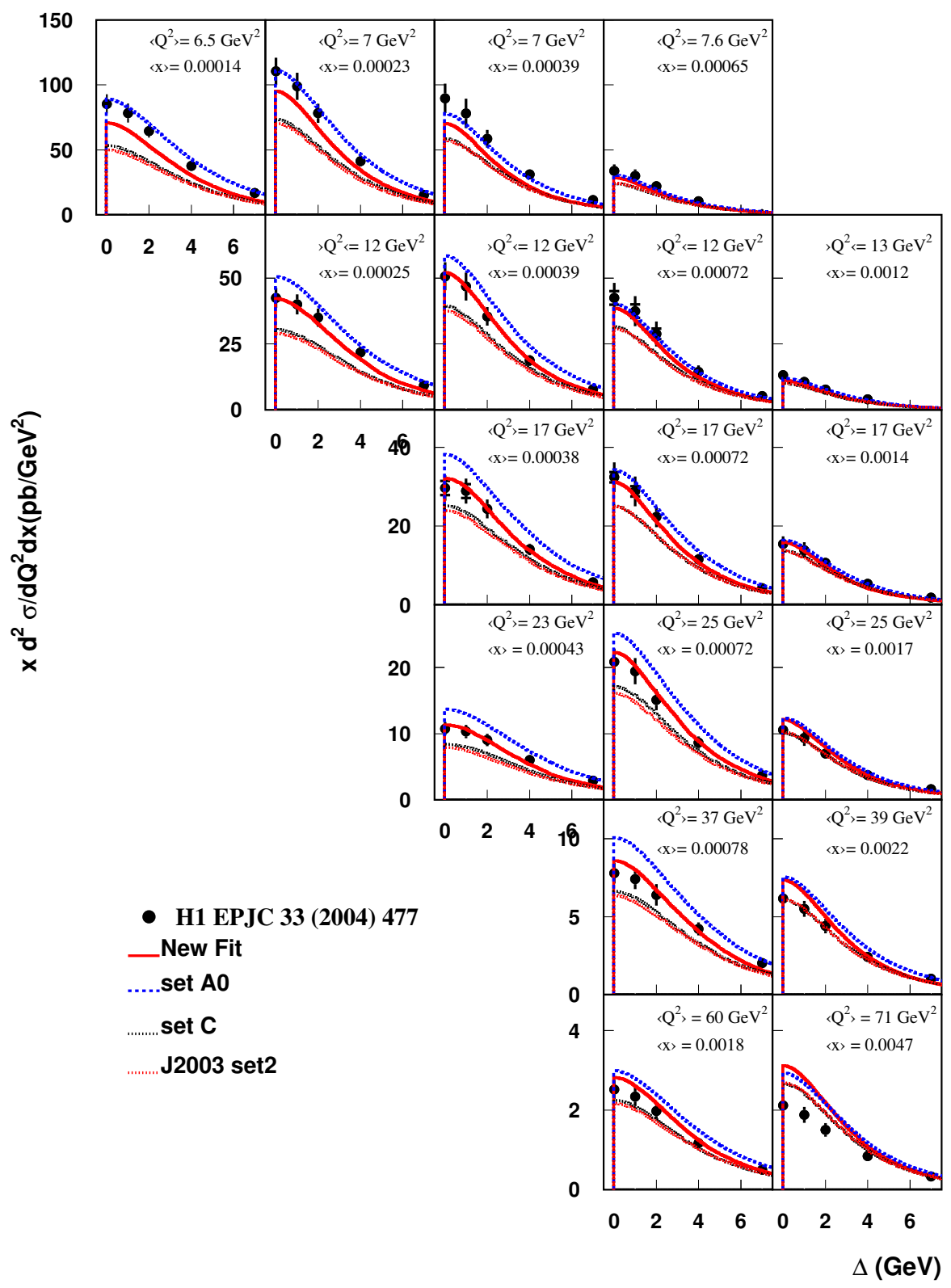

Figure 1: Di-jet data as a function of the difference between the transverse momentum requirement on the di-jets, $\Delta$, in bins of $x_{\mathrm{Bj}}$ and $Q^{2}$ compared to predictions from the CASCADE Monte Carlo event generator using the newly fitted PDF (full line) and 3 old PDFs; set A0 (dashed), set C(dotted/dark), J2003 set 2(dotted/light) . 
confirms that this is a minimum for $N$ and $B$, and $\mu$ has flattened out at 3. Varying this parameter around 3 thus not change the MC predictions.

The $\chi^{2} / n d f$ of the new fit is 2.1 , which should be compared to $\chi^{2} / n d f=3.5$ for the best performing old uGDF, which in this case is set A0. A uGDF that was determined from fits to the proton structure function [2]. There are two significant differences between these two uGDFs. The first one is the small $\mathrm{x}$ behaviour given by $B$, which is 0 for set A0, but suggested to be 0.25 by the fit to di-jet data. The second difference is that, while set A0 uses a non shifted Gaussian for the gluon $k_{t}$, i.e. $\mu=0$, the di-jet data suggests a large shift resulting in a decreasing starting distribution towards low $k_{t}$. The differences are illustrated in Fig. 3 where the uGDF is draw as a function of $x$ and $k_{t}^{2}$.
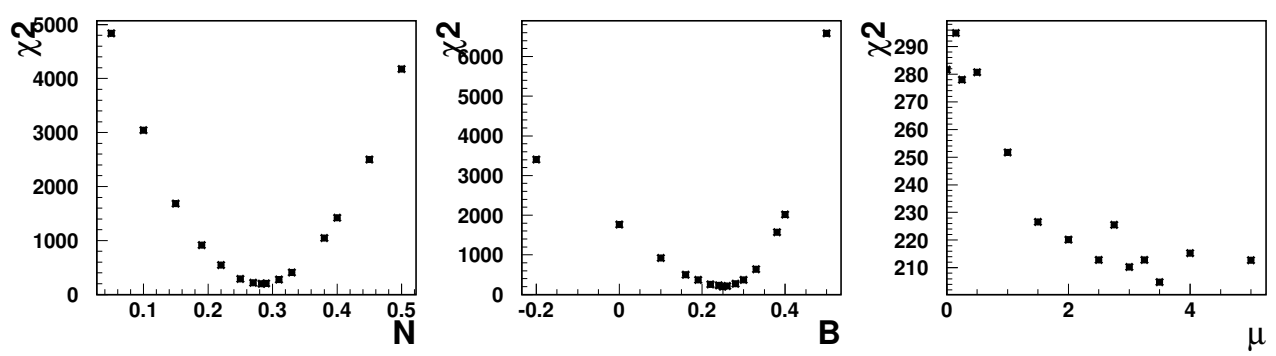

Figure 2: $\chi^{2}$ profiles as a function of the fitted parameters.
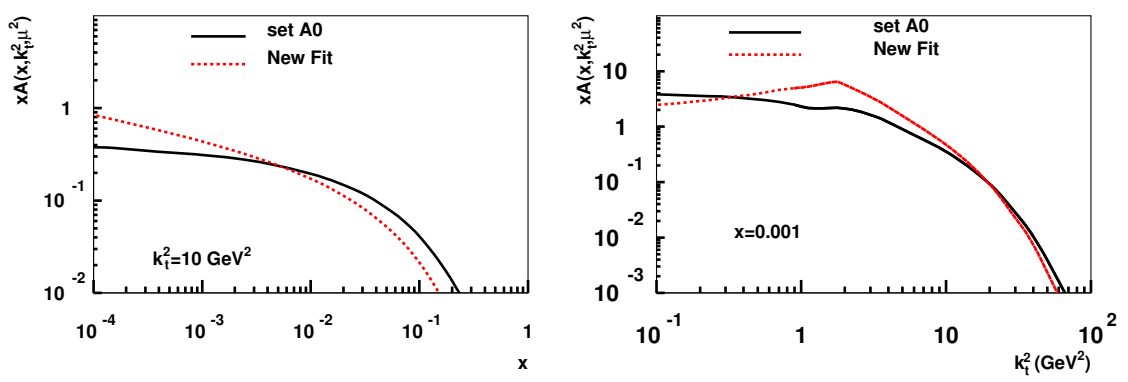

Figure 3: The newly fitted PDF (dashed line) compared to the old PDF set A0 (full line), drawn as a function of $x$ (for $\left.k_{t}^{2}=10 \mathrm{GeV}^{2}\right)$ and $k_{t}^{2}$ (for $\mathrm{x}=0.001$ ).

\section{References}

[1] P. Abreu et al. [DELPHI Collaboration], Z. Phys. C 73 (1996) 11.

[2] H. Jung, Comput. Phys. Commun. 143 (2002) 100 [arXiv:hep-ph/0109102].

[3] A. Aktas et al. [H1 Collaboration], Eur. Phys. J. C 33 (2004) 477 [arXiv:hep-ex/0310019].

[4] W. Press et al, Numerical Recipes, Cambridge University Press (1992), ISBN 0-521-43064-X

[5] Slides:

http: //indico. cern. $\mathrm{ch} /$ contributionDisplay $\cdot$ py? contribId $=137 \&$ session Id $=17 \& \operatorname{conf}$ Id $=24657$ 